\title{
Hilar Portion of the Right Hepatic Duct
}

National Cancer Institute

\section{Source}

National Cancer Institute. Hilar Portion of the Right Hepatic Duct. NCI Thesaurus. Code C38671.

The segment of the right hepatic duct located in the hilum of the liver. 